\title{
Negative feedback regulation of cellular antiviral signaling by RBCK1-mediated degradation of IRF3
}

\author{
Min Zhang ${ }^{1}$, Yang Tian ${ }^{1}$, Rui-Peng Wang ${ }^{1}$, Dong Gao ${ }^{1}$, Yan Zhang ${ }^{1}$, Fei-Ci Diao ${ }^{1}$, Dan-Ying Chen ${ }^{1}$, Zhong-He \\ Zhai ${ }^{1}$, Hong-Bing Shu ${ }^{2}$ \\ ${ }^{1}$ College of Life Sciences, Peking University, Beijing 100871, China; ${ }^{2}$ College of Life Sciences, Wuhan University, Wuhan 430072, \\ China
}

Viral infection causes host cells to produce type I interferons (IFNs), which are critically involved in viral clearance. Previous studies have demonstrated that activation of the transcription factor interferon regulatory factor (IRF) 3 is essential for virus-triggered induction of type I IFNs. Here we show that the E3 ubiquitin ligase RBCC protein interacting with PKC1 (RBCK1) catalyzes the ubiquitination and degradation of IRF3. Overexpression of RBCK1 negatively regulates Sendai virus-triggered induction of type I IFNs, while knockdown of RBCK1 has the opposite effect. Plaque assays consistently demonstrate that RBCK1 negatively regulates the cellular antiviral response. Furthermore, viral infection leads to induction of RBCK1 and subsequent degradation of IRF3. These findings suggest that the cellular antiviral response is controlled by a negative feedback regulatory mechanism involving RBCK1-mediated ubiquitination and degradation of IRF3.

Keywords: RBCK1, IRF3, antiviral response, type I IFNs, feedback regulation, E3 ligase

Cell Research (2008) 18:1096-1104. doi: 10.1038/cr.2008.277; published online 19 August 2008

\section{Introduction}

Host cells respond to viral infection by producing and secreting a family of cytokines called type I interferons (IFNs), including IFN $\beta$ and IFN $\alpha$ family members. These cytokines induce the expression of numerous downstream proteins that are collectively involved in the inhibition of viral replication [1-4]. Transcriptional regulation of the type I IFN genes requires the assembly of an enhanceosome that contains several transcription factors. For example, the IFN $\beta$ gene promoter contains a $\kappa \mathrm{B}$ site recognized by nuclear factor kappa B (NF- $\mathrm{KB})$, a site for ATF-2/c-Jun and two IFN-stimulated response elements (ISREs) recognized

Correspondence: Hong-Bing Shu ${ }^{\mathrm{a}}$, Dan-Ying Chen ${ }^{\mathrm{b}}$

${ }^{a}$ Tel: +86-27-68753795; Fax:+86-27-68753780

E-mail: shuh@whu.edu.cn

${ }^{\text {b}}$ Tel: +86-10-62757924; Fax: +86-10-62757924

E-mail: dychen@pku.edu.cn

Abbreviations: NF- $\mathrm{BB}$ (nuclear factor kappa B); IRF (interferon regulatory factor); ISRE (IFN-stimulated response elements); CARD (caspase recruitment domain); TBK1 (TANK-binding kinase 1); IKK $\varepsilon$ (inhibitor of nuclear factor $\kappa \mathrm{B}$ kinase subunit epsilon); RIG-I (retinoic acid-inducible gene I; MDA5 (melanoma differentiation associated protein-5); $\mathrm{SeV}$ (Sendai virus); RBCK1 (RBCC protein interacting with PKC1)

Received 17 March 2008; revised 14 May 2008; accepted 17 June 2008; published online 19 August 2008 by phosphorylated interferon regulatory factor (IRF) 3 or IRF7. Upon viral infection, these transcription factors are activated and cooperatively assemble into an enhanceosome on the IFN $\beta$ gene promoter, thereby activating the transcription of IFN $\beta[5,6]$.

The signaling pathways leading to the induction of type I IFNs have begun to emerge. Viral infection results in the accumulation of single- or double-stranded viral RNAs in the cytoplasm. The viral RNAs are recognized by two caspase recruitment domain (CARD)-containing RNA helicase proteins, retinoic acid-inducible gene I (RIG-I) and melanoma differentiation associated protein-5 (MDA5), which signal through a CARD-containing adapter protein called VISA by our group and MAVS, IPS-1, or Cardif by others [7-13]. Various studies have demonstrated that VISA plays a critical role in virus-induced NF- $\kappa \mathrm{B}$ activation, although it is controversial how this is achieved. On the other hand, it is widely accepted that VISA recruits TANKbinding kinase 1 (TBK1) and IRF3 to the RIG-I/MDA5 signaling complexes, in which IRF3 is phosphorylated by TBK1. Phosphorylated IRF3 dimerizes, forms a complex with $\mathrm{CBP} / \mathrm{p} 300$, and translocates into the nucleus to participate in the transcriptional activation of the type I IFN genes [14-17].

Type I IFNs are undetectable in cells under physi- 
ological conditions, but are promptly induced upon viral infection. To prevent autonomous production of type I IFNs or overproduction of these cytokines following viral infection, which causes a pathological immune response, the cell has evolved distinct strategies to tightly regulate their expression. It has been shown that LGP2, a helicase protein lacking CARDs at its $\mathrm{N}$-terminus, acts as a negative regulator by sequestering viral RNA from RIG-I [18-21]. The cellular protein DAK is associated with MDA5, and specifically inhibits MDA5-mediated, but not RIG-I-mediated, signaling [22]. The coiled-coil protein SIKE is associated with TBK1/inhibitor of nuclear factor kappa-B kinase subunit epsilon $(\mathrm{IKK} \varepsilon)$ and keeps these kinases inactive under physiological conditions [23].

Recent studies have demonstrated that post-translational modification by ubiquitin plays an important role in the regulation of virus-triggered type I IFN signaling. The dual-function deubiquitinating enzyme and ubiquitin ligase A20 negatively regulate RIG-I-induced antiviral signaling through an unidentified mechanism [24]. DUBA, a deubiquitinating enzyme, turns off expression of type I IFNs by deubiquitinating TRAF3, a signaling component in virus-triggered type I IFN signaling pathways [25]. The E3 ubiquitin ligase RNF125 targets RIG-I, MDA5 and VISA for degradation, and therefore negatively regulates type I IFN production [26]. In contrast, a recent study suggests that Lys 63-linked ubiquitination of RIG-I by the E3 ubiquitin ligase TRIM25 is crucial for the RIG-I-mediated cellular antiviral response [27].

RBCC protein interacting with PKC1 (RBCK1), also called HOIL-1 [28-30], XAP3 [31], or UIP28 [32], contains an N-terminal ubiquitin-like domain (UBL) and a C-terminal RING-IBR-RING (RBR) signature structure. It has been shown that RBCK1 is an E3 ubiquitin ligase for proteins involved in iron metabolism and for SOCS6 [28-30, 33]. Recently, we showed that RBCK1 negatively regulates TNF- and IL-1-induced NF- $\mathrm{KB}$ activation by targeting TAB2/3 for degradation [34]. In this report, we demonstrate that RBCK1 catalyzes the ubiquitination and degradation of IRF3, and negatively regulates virus-triggered type I IFN production and cellular antiviral response. In addition, we found that viral infection leads to induction of RBCK1 and subsequent degradation of IRF3. These findings suggest that the cellular antiviral response is controlled by a feedback negative regulatory mechanism involving RBCK1-mediated ubiquitination and degradation of IRF3.

\section{Results}

RBCK1 suppresses virus-triggered transcriptional activation of IFN $\beta$ and IFN 4
Previously, we demonstrated that RBCK1 negatively regulates TNF- and IL1-induced NF- $\kappa B$ activation by targeting $\mathrm{TAB} 2 / 3$ for degradation [34]. Since virus-triggered induction of type I IFNs involves NF- $\kappa B$ activation, we examined whether RBCK1 regulates virus-triggered signaling pathways. Reporter assays indicated that overexpression of RBCK1 dramatically inhibited Sendai virus (SeV)-induced transcriptional activation of IFN $\beta$ and IFN $\alpha 4$ gene promoters (Figure 1A). Furthermore, overexpression of RBCK1 inhibited SeV-induced expression of endogenous IFN $\beta$ and IFN $\alpha 4$ (Figure 1B).

To determine whether RBCK1 plays a negative regulatory role in virus-triggered type I IFN signaling under physiological conditions, we determined the effect of knockdown of RBCK1 by RNAi. We used two RBCK1 RNAi constructs in this study; one (\#4) significantly inhibited expression of RBCK1, while the other (\#1) had no detectable effect (Figure 1C). In reporter assays, knockdown of RBCK1 by the \#4 RNAi construct was sufficient to activate the IFN $\beta$ and IFN $\alpha 4$ promoters. In the same experiments, knockdown of RBCK1 potentiated SeV-induced activation of the IFN $\beta$ and IFN $\alpha 4$ promoters (Figure 1D). In addition, knockdown of RBCK1 also induced or potentiated expression of endogenous IFN $\beta$ and IFN $\alpha 4$ in the absence or presence of viral stimulation (Figure 1E). These data suggest that RBCK1 is a physiological suppressor of virus-triggered type I IFN signaling.

RBCK1 negatively regulates the cellular antiviral response

Since RBCK1 is a physiological suppressor of virus-triggered type I IFN signaling, we determined whether RBCK1 plays a role in the cellular antiviral response. Previously, it was shown that wild-type VSV induces little expression of type I IFNs in 293 cells, while overexpression of RIGI-N, MDA5-N, VISA, or TBK1 can induce transcription of type I IFNs, and causes a cellular antiviral response $[7-13,16,17]$. Accordingly, we tested whether RBCK1 affects the cellular antiviral response mediated by these signaling proteins in 293 cells. Using plaque assays, we found that overexpression of RBCK1 was able to significantly antagonize the inhibitory effect on viral replication mediated by RIG-I-N, MDA5-N, VISA, or TBK1 (Figure 2A). Consistently, knockdown of RBCK1 inhibited viral replication (Figure 2B). These results suggest that RBCK1 acts as a physiological inhibitor of the cellular antiviral response.

RBCK1 negatively regulates virus-triggered IFN signaling by destabilizing IRF3

To determine the molecular mechanisms responsible for RBCK1-mediated inhibition of virus-triggered type I IFN 
A

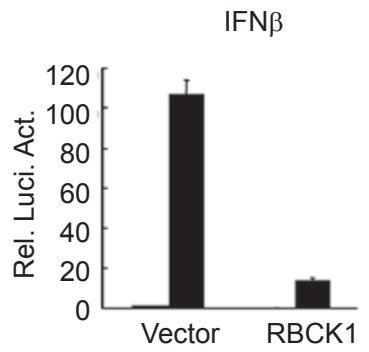

B

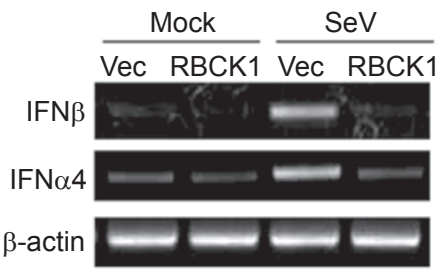

D

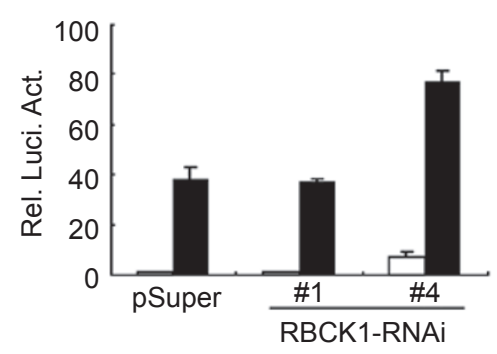

E

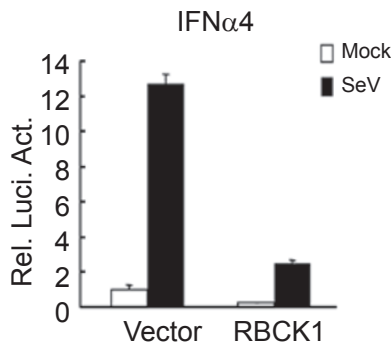

C

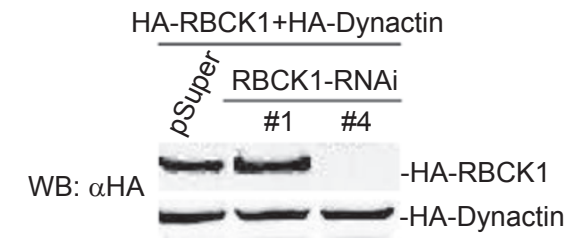

WB

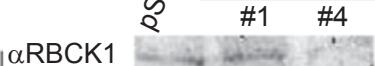

$\left.\right|^{\alpha \mathrm{RBCK} 1}$

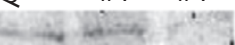

$\alpha \mathrm{GAPDH}$

IFN $\alpha 4$

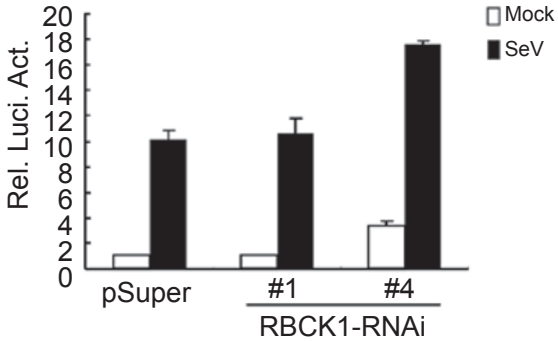

Figure 1 RBCK1 regulates SeV-triggered type I IFN signaling. (A) RBCK1 inhibits SeV-induced activation of IFN and IFNa4 promoters. The 293 cells $\left(\sim 5 \times 10^{4}\right)$ were transfected with IFN $\beta$ or IFN $\alpha 4$ promoter luciferase plasmid (50 ng), pRL-TK Renilla luciferase plasmid (50 ng), and an expression vector for Flag-RBCK1 $(0.1 \mu \mathrm{g})$ or an empty vector $(0.1 \mu \mathrm{g})$. At $12 \mathrm{~h}$ after transfection, cells were infected with SeV (filled bars) or left uninfected (open bars) for $12 \mathrm{~h}$ before luciferase assays were performed. (B) RBCK1 inhibits SeV-induced expression of endogenous IFN $\beta$ and IFN $\alpha 4$. The 293 cells $\left(2 \times 10^{5}\right)$ were transfected with an expression vector for Flag-RBCK1 $(0.5 \mu \mathrm{g})$ or an empty vector $(0.5 \mu \mathrm{g})$. At $12 \mathrm{~h}$ after transfection, cells were infected with $\mathrm{SeV}$ or left uninfected for $12 \mathrm{~h}$ before RT-PCR analysis of the indicated mRNAs. (C) Effects of RBCK1 RNAi plasmids on the expression of overexpressed and endogenous RBCK1. The 293 cells $\left(2 \times 10^{5}\right)$ were transfected with HA-tagged RBCK1 and Dynactin (upper panels) or empty control plasmid (lower panels) $(1 \mu \mathrm{g}$ each), together with pSuper control, RBCK1-RNAi-\#1 or RBCK1-RNAi-\#4 plasmid (2 $\mathrm{gg}$ each) as indicated. At $48 \mathrm{~h}$ after transfection, cell lysates were analyzed by Western blot with anti-HA (upper panels), anti-RBCK1, or anti-GAPDH (lower panels) antibody. (D) Knockdown of RBCK1 potentiates SeV-triggered activation of IFN $\beta$ and IFN $\alpha 4$ promoters in 293 cells. The 293 cells $\left(\sim 5 \times 10^{4}\right)$ were transfected with the IFN $\beta$ or IFN $\alpha 4$ promoter reporter plasmids (50 ng) and the indicated RBCK1 RNAi plasmid (1 $\mu \mathrm{g})$. At $36 \mathrm{~h}$ after transfection, cells were infected with SeV or left uninfected for $12 \mathrm{~h}$ before luciferase assays. (E) knockdown of RBCK1 increased SeV-triggered expression of endogenous IFN $\beta$ and IFN $\alpha 4$. The 293 cells $\left(2 \times 10^{5}\right)$ were transfected with a control or the indicated RBCK1 RNAi plasmid $(2$ $\mu \mathrm{g}$ each). Thirty-six hours after transfection, cells were infected with SeV or left uninfected for $12 \mathrm{~h}$ before RT-PCR analysis of the expression of the indicated mRNAs. 
A

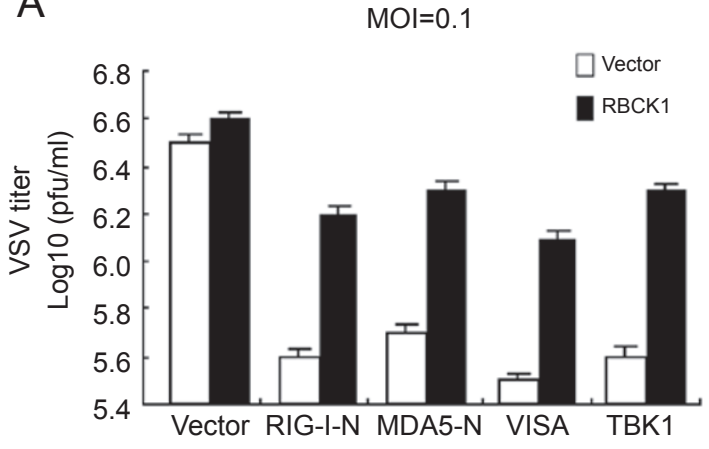

B

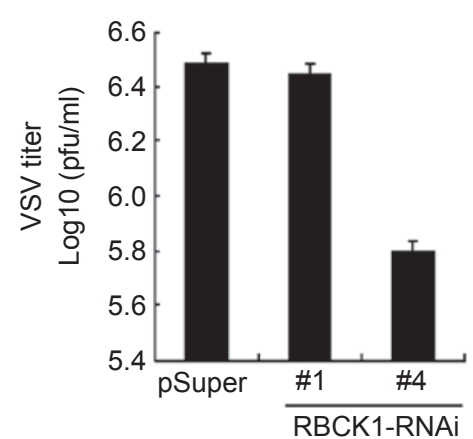

Figure 2 Effect of overexpression or knockdown of RBCK1 on the cellular antiviral response. (A) Overexpression of RBCK1 inhibits RIG-I-N-, MDA5-N-, VISA- or TBK1-mediated cellular antiviral response. The 293 cells $\left(2 \times 10^{5}\right)$ were transfected with the indicated expression plasmids ( $1 \mu \mathrm{g}$ each). Twenty-four hours after transfection, cells were infected with VSV [multiplicity of infection $(\mathrm{MOI})=0.1$ ], and supernatants were harvested at 12 $\mathrm{h}$ postinfection and analyzed for VSV production by standard plaque assays. (B) Knockdown of RBCK1 enhances the cellular antiviral response. The 293 cells $\left(2 \times 10^{5}\right)$ were transfected with a control or the indicated RBCK1 RNAi plasmid ( $2 \mu \mathrm{g}$ each). Thirtysix hours after transfection, cells were infected with VSV $(\mathrm{MOI}=$ 0.1 ), and supernatants were harvested at $12 \mathrm{~h}$ postinfection for plaque assays.

signaling, we examined whether RBCK1 inhibits virustriggered NF- $\mathrm{KB}$ and IRF3 activation. As shown in Figure 3A, RBCK1 significantly inhibited both $\mathrm{SeV}$-induced $\mathrm{NF}-\mathrm{\kappa B}$ and ISRE activation. Previously, we demonstrated that RBCK1 targets $\mathrm{TAB} 2 / 3$ for degradation and therefore inhibits TNF- and IL1-induced NF- $\mathrm{BB}$ activation [34]. Since TAK1, a kinase associated with TAB2/3, is involved in $\mathrm{SeV}$-induced NF- $\mathrm{KB}$ [11], it is possible that RBCK1 inhibits SeV-induced NF- $\kappa \mathrm{B}$ activation by targeting TAB2/3. Therefore, we focused on the mechanism of RBCK1-mediated inhibition of ISRE. To determine which signaling components in the virus-triggered IRF3 activation pathway are targeted by RBCK1, we determined the effect of RBCK1 on ISRE activation mediated by the involved signaling components. In reporter assays, RBCK1 had a significant inhibitory effect on ISRE activation triggered by overexpression of RIG-I-N, MDA5-N, VISA, TBK1, and IRF3, but not IRF7 (Figure 3B), suggesting that RBCK1 functions specifically at or downstream of IRF3.

We next sought to identify the relationship between RBCK1 and IRF3. Interestingly, the protein level of IRF3, but not IRF7, was dramatically down-regulated when it was coexpressed with RBCK1 (Figure 3C). In addition, overexpression of RBCK1 caused down-regulation of endogenous IRF3, and this effect was reversed by MG132, a proteasome inhibitor (Figure 3D). Moreover, knockdown of RBCK1 by RNAi caused an increased level of endogenous IRF3 (Figure 3E). Taken together, these data suggest that RBCK1 targets IRF3 for degradation by the proteasomes.

RBCK1 physically interacts with and ubiquitinates IRF3

Since RBCK1 targets IRF3 for degradation in a proteasome-dependent manner, we determined whether RBCK physically interacts with and ubiquitinates IRF3. In transient transfection and coimmunoprecipitation experiments, RBCK1 interacted with IRF3 in the presence of MG132 (Figure 4A). Moreover, the N-terminal UBL domain of RBCK1, RBCK1 (1-220), was sufficient for its interaction with IRF3, whereas an interaction between IRF3 and the C-terminal RBR domain of RBCK1, RBCK1 (221-510), was undetectable (Figure 4B). In reporter assays, RBCK1 (221-510) inhibited SeV-induced ISRE activation as potently as did full-length RBCK1, while RBCK1 (1-220) inhibited SeV-induced ISRE activation to a lesser degree (Figure 4C). Taken together, these data suggest that RBCK1 physically interacts with IRF3 via its UBL domain, and destabilizes IRF3 via the RBR domain.

Since RBCK1 is an E3 ubiquitin ligase and causes IRF3 degradation, we determined whether RBCK1 ubiquitinates IRF3. We transfected 293 cells with expression plasmids for Flag-IRF3, HA-ubiquitin, and HA-RBCK1. Coimmunoprecipitation and western blot analysis indicated that RBCK1 significantly polyubiquitinated IRF3 (Figure 4D).

\section{RBCK1 is up-regulated following viral infection}

Having established that RBCK1 negatively regulates innate antiviral signaling by targeting IRF3 for degradation, we next examined whether viral infection affects RBCK1 expression. We found that endogenous RBCK1 was up-regulated following $\mathrm{SeV}$ infection in a time-dependent manner (Figure 5A). Interestingly, SeV-induced up-regulation of RBCK1 was accompanied by a temporal reduction of endogenous IRF3 (Figure 5A). Knockdown of RBCK1 by RNAi rescued SeV-induced IRF3 down-regulation (Figure 5B). These observations suggest that RBCK1 
A

$\mathrm{NF}-\mathrm{kB}$

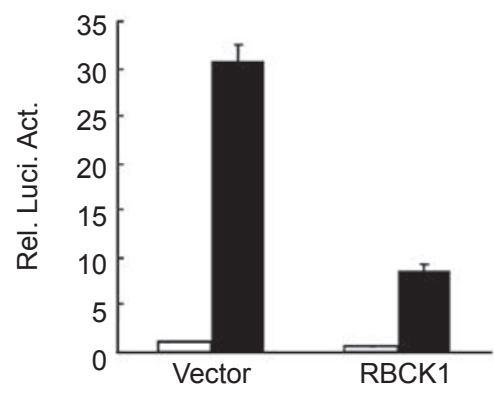

B

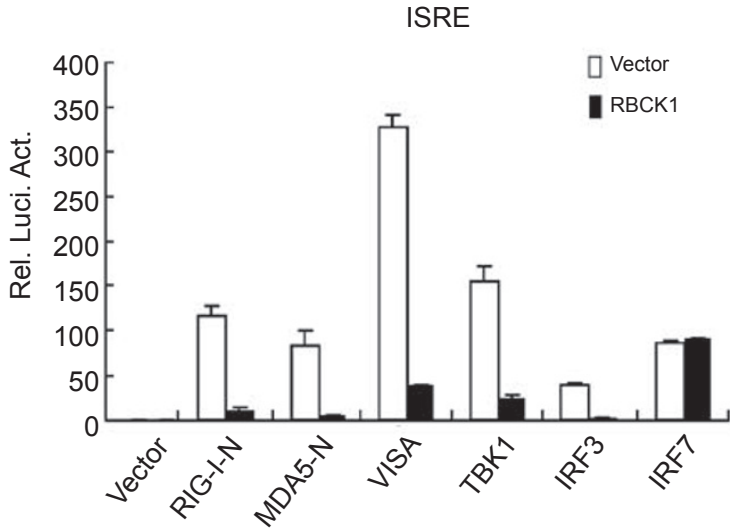

D

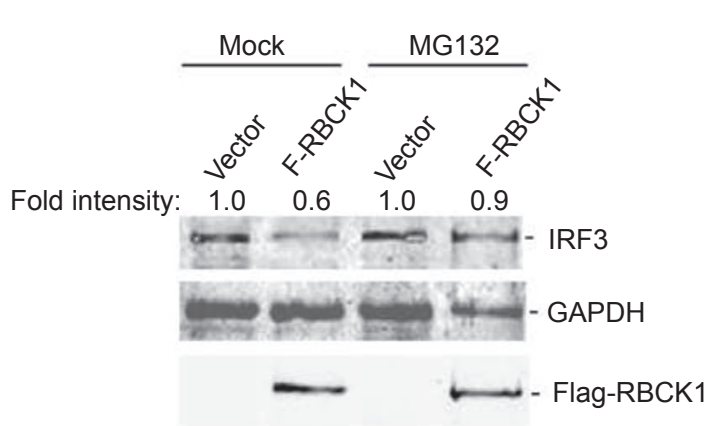

ISRE

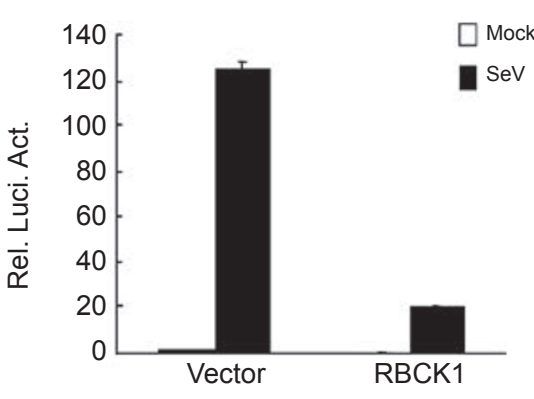

C

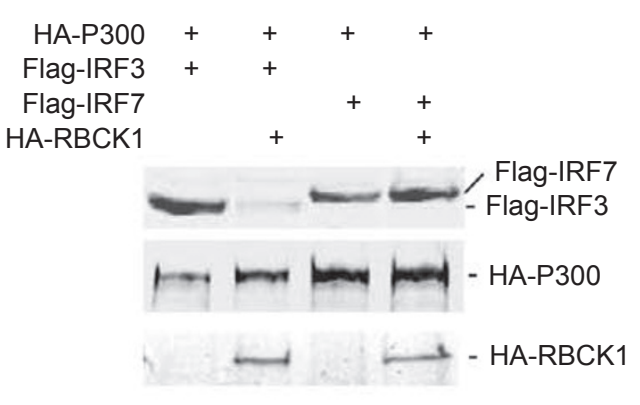

E

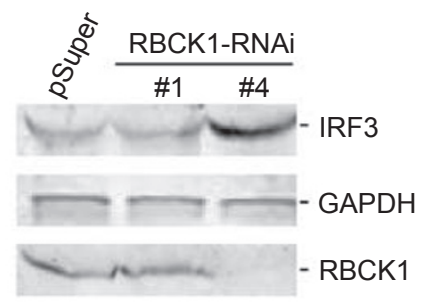

Figure 3 RBCK1 inhibits virus-triggered IFN signaling by targeting IRF3 for degradation. (A) RBCK1 inhibits SeV-triggered NF-kB and ISRE activation. The 293 cells $\left(\sim 5 \times 10^{4}\right)$ were transfected with NF-kB or ISRE luciferase plasmid $(50 \mathrm{ng}), \mathrm{pRL}-\mathrm{TK}$ Renilla luciferase plasmid $(50 \mathrm{ng})$, and an expression vector for Flag-RBCK1 $(0.1 \mu \mathrm{g})$ or an empty vector $(0.1 \mu \mathrm{g})$. At $12 \mathrm{~h}$ after transfection, cells were infected with SeV (filled bars) or left uninfected (open bars) for $12 \mathrm{~h}$ before luciferase assays were performed. (B) RBCK1 inhibits ISRE activation mediated by RIG-I-N, MDA5-N, VISA, TBK1, and IRF3, but not IRF7. The 293 cells $\left(\sim 5 \times 10^{4}\right)$ were transfected with ISRE luciferase plasmid $(50 \mathrm{ng})$, pRL-TK Renilla luciferase plasmid $(50 \mathrm{ng})$, and the indicated plasmids $(0.1 \mu \mathrm{g}$ each). At $24 \mathrm{~h}$ after transfection, luciferase assays were performed. (C) RBCK1 specifically destabilizes IRF3. The 293 cells $\left(2 \times 10^{5}\right)$ were transfected with the indicated expression plasmids $(1 \mu \mathrm{g}$ each). At $24 \mathrm{~h}$ after transfection, cell lysates were analyzed by western blot with anti-Flag (upper panel) or anti-HA (middle and lower panels) antibody. (D) RBCK1 causes degradation of endogenous IRF3 in a proteasome-dependent manner. The 293 cells $\left(2 \times 10^{5}\right)$ were transfected with an expression vector for Flag-RBCK1 or an empty vector $(1 \mu \mathrm{g}$ each). At $24 \mathrm{~h}$ after transfection, cells were treated with MG132 $(10 \mu \mathrm{M})$ or left untreated for $6 \mathrm{~h}$ before Western blot analysis was performed with anti-IRF3 (upper panel), anti-GAPDH (middle panel), or anti-Flag (lower panel). (E) Knockdown of RBCK1 up-regulates endogenous IRF3. The 293 cells were transfected as in Figure 1C. At $48 \mathrm{~h}$ after transfection, cell lysates were analyzed by Western blot with anti-IRF3 (upper panel), anti-GAPDH (middle panel), or anti-RBCK1 (lower panel) antibody. 
A

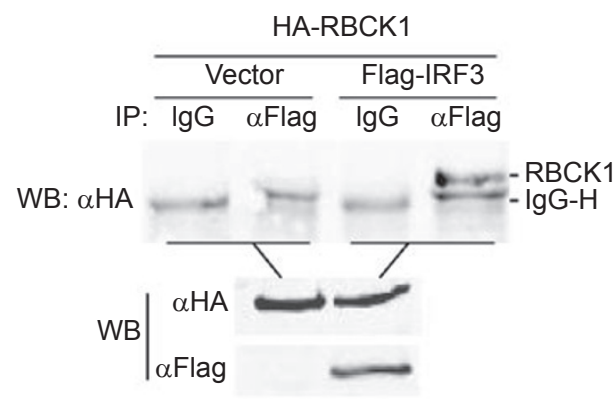

B

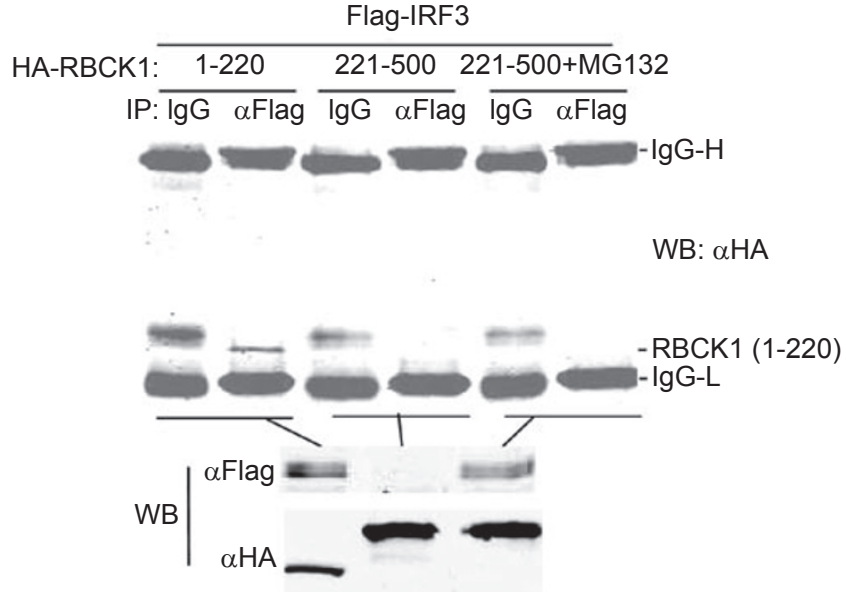

D

$\frac{\text { F-IRF3+HA-ubiquitin }}{\text { IP: } \frac{\text { Vector }}{\text { IgG } \alpha \text { Flag }} \frac{\text { HA-RBCK1 }}{\lg \quad \alpha \text { Flag }}}$

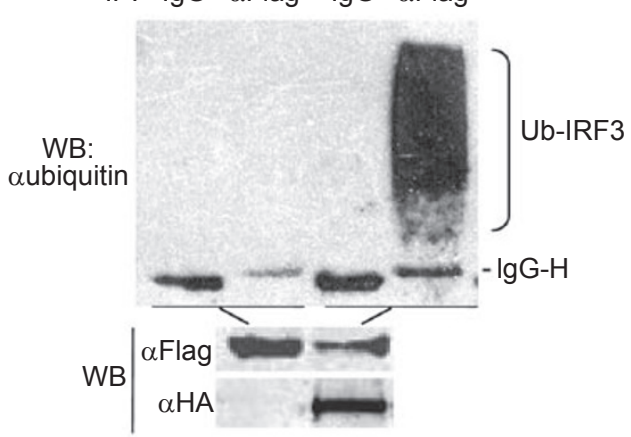

Figure 4 RBCK1 interacts with and ubiquitinates IRF3. (A) RBCK1 interacts with IRF3. The 293 cells $\left(2 \times 10^{6}\right)$ were transfected with the indicated plasmids ( $3 \mu \mathrm{g}$ each). At $18 \mathrm{~h}$ after transfection, cells were treated with MG132 $(10 \mu \mathrm{M})$ for $6 \mathrm{~h}$ before cell lysates were immunoprecipitated with anti-Flag antibody ( $\alpha$ Flag) or control mouse lgG (mlgG). The immunoprecipitates were analyzed by western blot with anti-HA antibody (upper panel). Expression of the transfected proteins was analyzed by western blot with anti-Flag or anti-HA antibody (middle and lower panels). (B) Domain mapping of the interaction between RBCK1 and IRF3. The 293 cells $\left(2 \times 10^{6}\right)$ were transfected with the indicated plasmids $(3 \mu \mathrm{g}$ each) and coimmunoprecipitation experiments were performed as in (A). (C) Effects of RBCK1 mutants on SeV-triggered ISRE activation. The 293 cells $\left(\sim 5 \times 10^{4}\right)$ were transfected with ISRE luciferase plasmid (50 ng), pRL-TK Renilla luciferase plasmid $(50 \mathrm{ng})$, and the indicated plasmids $(0.1 \mu \mathrm{g})$. At $12 \mathrm{~h}$ after transfection, cells were infected with $\mathrm{SeV}$ (filled bars) or left uninfected (open bars) for $12 \mathrm{~h}$ before luciferase assays were performed. (D) RBCK1 ubiquitinates IRF3. The 293 cells $\left(2 \times 10^{6}\right)$ were transfected with expression plasmids for Flag-IRF3, HA-ubiquitin, HA-RBCK1, or control plasmid ( $3 \mu \mathrm{g}$ each). At $18 \mathrm{~h}$ after transfection, cells were treated with MG132 (10 $\mu \mathrm{M})$ for $6 \mathrm{~h}$. Cell lysates were immunoprecipitated with anti-Flag antibody ( $\alpha$ Flag) or control mouse $\lg$ ( $(\mathrm{mlgG})$. The immunoprecipitates were analyzed by western blot with anti-ubiquitin antibody (upper panel). Expression of the transfected proteins was analyzed by western blot with anti-Flag or anti-HA antibody (middle and lower panels).

regulates the innate antiviral response through a negative feedback mechanism.

\section{Discussion}

Viral infection results in transcriptional induction of type I IFNs and other cytokines that have inhibitory effects on viral replication in infected and neighboring uninfected cells. However, uncontrolled excessive production of type I IFNs significantly contributes to chronic inflammation and autoimmune diseases, underscoring the importance of negative regulation of type I IFN [35-38]. The host innate immune system has developed distinct strategies to ensure proper production of type I IFNs following viral infection 


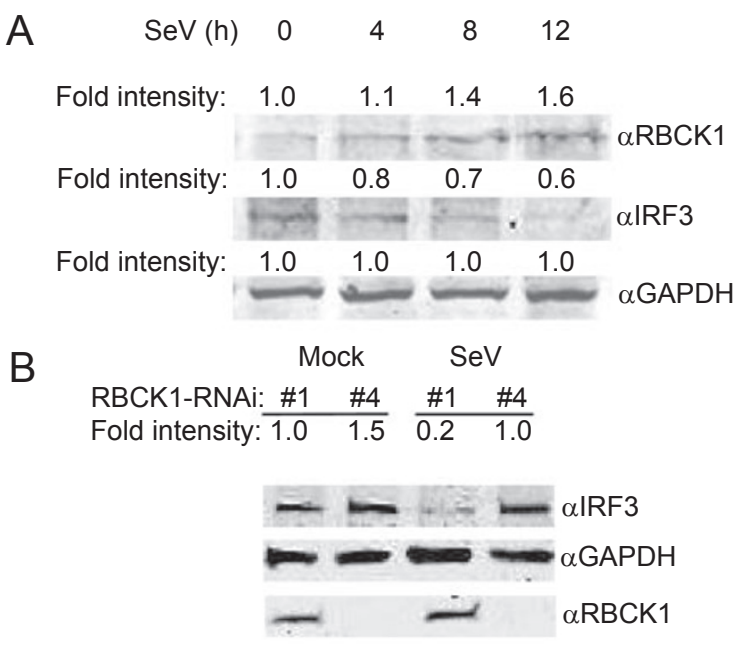

Figure 5 RBCK1 mediates SeV-induced IRF3 down-regulation. (A) Expression of RBCK1 is induced by SeV. The 293 cells were infected by $\mathrm{SeV}$ for the indicated times before cell lysates were analyzed by Western blot with anti-RBCK1 (upper panel), anti-IRF3 (middle panel), or anti-GAPDH (lower panel). (B) Knockdown of RBCK1 rescues SeV-induced down-regulation of IRF3. The 293 cells were transfected with the indicated RBCK1 RNAi plasmids. At $36 \mathrm{~h}$ after transfection, cells were infected with $\mathrm{SeV}$ for $12 \mathrm{~h}$ or left untreated. Cell lysates were analyzed by Western blot with the indicated antibodies.

[18-26]. In the present work, we identified the E3 ubiquitin ligase RBCK1 as a negative feedback regulator of the cellular antiviral response.

Our results demonstrated that overexpression of RBCK1 significantly inhibits $\mathrm{SeV}$-induced expression of type I IFNs, while knockdown of RBCK1 induces or potentiates SeV-induced expression of type I IFNs (Figure 1). In analyzing the target of RBCK1 in virus-induced type I IFN signaling pathways, we found that RBCK1 interacts with and ubiquitinates IRF3 and causes its degradation through a proteasome-dependent manner, while knockdown of RBCK1 by RNAi causes an increased level of IRF3 (Figures 3 and 4). Although IRF3 shares a similar structure with IRF7, RBCK1 destabilizes IRF3 but not IRF7, suggesting that RBCK1 specifically regulates IRF3-dependent signaling. Domain mapping experiments suggest that the $\mathrm{N}$-terminal UBL domain of RBCK1 is sufficient for its interaction with IRF3, whereas the C-terminal RBR domain is responsible for degrading IRF3 and inhibiting $\mathrm{SeV}$-induced ISRE activation (Figure 4B). Although the N-terminal UBL domain lacks E3 ubiquitin ligase activity, it weakly inhibited $\mathrm{SeV}$-induced ISRE activation in reporter assays (Figure 4C). This effect is probably caused by its recruitment of endogenous RBCK1 to IRF3. The C-terminal RBR domain of RBCK1, RBCK1 (221-510), potently induced degradation of IRF3 and inhibited SeV-induced ISRE ac- tivation, even though it did not interact with IRF3 (Figure 4B and $4 \mathrm{C}$ ). The simplest explanation for this observation is that our coimmunoprecipitation experiments were not sensitive enough to detect their interaction.

Our studies indicate that viral infection induces expression of RBCK1, which is accompanied by down-regulation of IRF3. In addition, virus-induced IRF3 down-regulation is rescued by knockdown of RBCK1 (Figure 5). Taken together, these data suggest that the E3 ubiquitin ligase RBCK 1 targets IRF3 for degradation and regulates virusinduced type I IFN signaling through a negative feedback regulatory mechanism.

Previously, various studies have demonstrated that IRF3 degradation following virus infection plays a significant role in the termination of the IRF3-dependent cellular antiviral response [14, 39-44]. These studies have identified several cellular and viral proteins that are involved in IRF3 ubiquitination and degradation, including an unidentified Cullin-based ubiquitin ligase [41], the peptidyl-prolyl isomerase Pin1 [42], ICP0 of herpesvirus 1 [39, 40, 43], and NSP1 of rotavirus [44]. However, a bona fide cellular E3 ubiquitin ligase for IRF3 is unknown. Our discovery that RBCK1 is a virus-inducible E3 ubiquitin ligase for IRF3 ubiquitination and degradation resolves the molecular mechanism accounting for IRF3 degradation-dependent control of excessive cellular antiviral response.

\section{Materials and Methods}

\section{Reagents and antibodies}

Mouse monoclonal antibodies against Flag (M2) and HA epitopes (Sigma, St Louis, USA), rabbit polyclonal antibodies against IRF3 (SC-9082) and ubiquitin (SC-9133) (Santa Cruz Biotechnology, Santa Cruz, USA), VSV (Dr Hong-Kui Deng, Peking University, Beijing, China) and SeV (Dr Cong-Yi Zheng, Wuhan University, Wuhan, China) were obtained from the indicated sources. Mouse anti-RBCK1 antiserum was described previously [34].

\section{Constructs}

Mammalian expression plasmids for Flag-RIG-I, Flag-MDA5, Flag-VISA, Flag-TBK1, Flag-IRF3, Flag-IRF7, Flag- or HA-tagged RBCK1 and RBCK1 deletion mutants were described previously [11, 22, 23]. Mammalian expression plasmid for HA-P300 was provided by Dr David Livingston (Dana Farber Cancer Institute, Boston, USA). ISRE-luciferase reporter plasmid was purchased from Stratagene (West Cedar Creek, USA). NF- $\kappa B$ and IFN $\beta$ promoter luciferase reporter plasmids were described previously [11]. IFNa4-luciferase reporter plasmid was provided by Dr Zheng-Fan, Jiang (Peking University, Peking, China).

\section{Transfection and reporter gene assays}

293 cells $\left(\sim 5 \times 10^{4}\right)$ were seeded on 24 -well dishes and transfected the following day by standard calcium phosphate precipitation. To normalize for transfection efficiency, $0.1 \mathrm{mg}$ of pRL-TK Renilla luciferase reporter plasmid was added to each transfection. Ap- 
proximately $20 \mathrm{~h}$ after transfection, luciferase assays were performed using a dual-specific luciferase assay kit (Promega, Madison, WI). Firefly luciferase activities were normalized based on Renilla luciferase activities. All reporter gene assays were repeated at least three times. Data shown are average values \pm s.d. from one representative experiment.

\section{$R T-P C R$}

Total RNA was isolated from 293 cells using Trizol reagent (Tiangen Bio-Tech, China). cDNA was made from total RNA using M-MLV (Promega, USA) according to the manufacturer's instructions. Gene-specific primer sequences were as follows.

IFN- $\beta$ : 5'-CAC GAC AGC TCT TTC CAT GA-3' (forward), 5'AGC CAG TGC TCG ATG AAT CT-3' (reverse);

IFN $\alpha$ 4: 5'-CCC GAG GAG GAG TTT GAT-3' (forward), 5'-TTC CAG GTC ATT CAG TTG C-3' (reverse);

$\beta$-actin: 5'-GTC GTC GAC AAC GGC TCC GGC ATG-3' (forward), 5'-ATT GTA GAA GGT GTG GTG CCA GAT-3' (reverse).

\section{RNAi experiments}

The experiments were performed as described [34].

\section{VSV plaque assay}

293 cells grown in media containing 1\% FBS were incubated with VSV at MOI 0.1 for $1 \mathrm{~h}$ before replacement with the complete media containing $10 \% \mathrm{FBS}$. After $24 \mathrm{~h}$, the supernatant was harvested and diluted to infect confluent BHK21 cells cultured on 24-well dishes. At $1 \mathrm{~h}$ postinfection, the supernatant was removed and $2 \%$ methylcellulose was overlaid. On day 3 postinfection, the overlay was removed and cells were fixed with $0.5 \%$ glutaraldehyde for 30 min and stained with $1 \%$ crystal violet in $70 \%$ methanol for 15 min. Plaques were counted, averaged, and multiplied by the dilution factor to determine viral titer as $\log 10(\mathrm{pfu} / \mathrm{ml})$.

\section{Coimmunoprecipitation and western blot analysis}

For transient transfection and coimmunoprecipitation experiments, 293 cells $\left(\sim 1 \times 10^{6}\right)$ were transfected for $24 \mathrm{~h}$. The transfected cells were lysed in $1 \mathrm{ml}$ of lysis buffer (20 mM Tris, $\mathrm{pH} 7.5,150$ $\mathrm{mM} \mathrm{NaCl}, 1 \%$ Triton, $1 \mathrm{mM}$ EDTA, $10 \mu \mathrm{g} / \mathrm{ml}$ aprotinin, $10 \mu \mathrm{g} / \mathrm{ml}$ leupeptin, $1 \mathrm{mM}$ phenylmethylsulfonyl fluoride). For each immunoprecipitation, a $0.4 \mathrm{ml}$ aliquot of lysate was incubated for $2 \mathrm{~h}$ with 0.5 $\mu \mathrm{g}$ of the indicated antibody or control IgG and $25 \mu 1$ of $1: 1$ slurry of GammaBind G Plus-Sepharose (GE Healthcare). The Sepharose beads were washed three times with $1 \mathrm{ml}$ of lysis buffer containing $500 \mathrm{mM} \mathrm{NaCl}$. The precipitates were analyzed by western blots as described [11].

\section{Acknowledgments}

We thank members of our laboratory for technical help and stimulating discussion. This work was supported by the National Basic Research Program of China (No. 2006CB504301) and the National Natural Science Foundation of China (No. 30630019 and No. 30570959).

\section{References}

1 Darnell JE Jr, Kerr IM, Stark GR. Jak-STAT pathways and tran- scriptional activation in response to IFNs and other extracellular signaling proteins. Science 1994; 264:1415-1421.

2 Siegal FP, Kadowaki N, Shodell M, et al. The nature of the principal type 1 interferon-producing cells in human blood. Science 1999; 284:1835-1837.

3 Levy DE, Garcia-Sastre A. The virus battles: IFN induction of the antiviral state and mechanisms of viral evasion. Cytokine Growth Factor Rev 2001; 12:143-156.

4 Theofilopoulos AN, Baccala R, Beutler B, Kono DH. Type I interferons (alpha/beta) in immunity and autoimmunity. Annu Rev Immunol 2005; 23:307-336.

5 Au WC, Moore PA, Lowther W, Juang YT, Pitha PM. Identification of a member of the interferon regulatory factor family that binds to the interferon-stimulated response element and activates expression of interferon-induced genes. Proc Natl Acad Sci USA 1995; 92:11657-11661.

6 Wathelet MG, Lin CH, Parekh BS, et al. Virus infection induces the assembly of coordinately activated transcription factors on the IFN-beta enhancer in vivo. Mol Cell 1998; 1:507-518.

7 Yoneyama M, Kikuchi M, Natsukawa T, et al. The RNA helicase RIG-I has an essential function in double-stranded RNA-induced innate antiviral responses. Nat Immunol 2004; 5:730-737.

8 Meylan E, Curran J, Hofmann K, et al. Cardif is an adaptor protein in the RIG-I antiviral pathway and is targeted by hepatitis C virus. Nature 2005; 437:1167-1172.

9 Seth RB, Sun L, Ea CK, Chen ZJ. Identification and characterization of MAVS, a mitochondrial antiviral signaling protein that activates NF-kappaB and IRF 3. Cell 2005; 122:669-682.

10 Kawai T, Takahashi K, Sato S, et al. IPS-1, an adaptor triggering RIG-I- and Mda5-mediated type I interferon induction. Nat Immunol 2005; 6:981-988.

$11 \mathrm{Xu} \mathrm{LG}$, Wang YY, Han KJ, et al. VISA is an adapter protein required for virus-triggered IFN-beta signaling. Mol Cell 2005; 19:727-740.

12 Kawai T, Akira S. Innate immune recognition of viral infection. Nat Immunol 2006; 7:131-137.

13 Sun Q, Sun L, Liu HH, et al. The specific and essential role of MAVS in antiviral innate immune responses. Immunity 2006; 24:633-642.

14 Lin R, Heylbroeck C, Pitha PM, Hiscott J. Virus-dependent phosphorylation of the IRF-3 transcription factor regulates nuclear translocation, transactivation potential, and proteasome-mediated degradation. Mol Cell Biol 1998; 18:2986-2996.

15 Hiscott J, Pitha $\mathrm{P}$, Genin $\mathrm{P}$, et al. Triggering the interferon response: the role of IRF-3 transcription factor. $J$ Interferon Cytokine Res 1999; 19:1-13.

16 Fitzgerald KA, McWhirter SM, Faia KL, et al. IKKepsilon and TBK1 are essential components of the IRF3 signaling pathway. Nat Immunol 2003; 4:491-496.

17 Sharma S, tenOever BR, Grandvaux N, et al. Triggering the interferon antiviral response through an IKK-related pathway. Science 2003; 300:1148-1151.

18 Rothenfusser S, Goutagny N, DiPerna G, et al. The RNA helicase Lgp2 inhibits TLR-independent sensing of viral replication by retinoic acid-inducible gene-I. J Immunol 2005; 175:52605268 .

19 Yoneyama M, Kikuchi M, Matsumoto K, et al. Shared and unique functions of the DExD/H-box helicases RIG-I, MDA5, and LGP2 in antiviral innate immunity. J Immunol 2005; 175:2851-2858. 
20 Komuro A, Horvath CM. RNA- and virus-independent inhibition of antiviral signaling by RNA helicase LGP2. J Virol 2006; 80:12332-12342.

21 Saito T, Hirai R, Loo YM, et al. Regulation of innate antiviral defenses through a shared repressor domain in RIG-I and LGP2. Proc Natl Acad Sci USA 2007; 104:582-587.

22 Diao F, Li S, Tian Y, et al. Negative regulation of MDA5- but not RIG-I-mediated innate antiviral signaling by the dihydroxyacetone kinase. Proc Natl Acad Sci USA 2007; 104:11706-11711.

23 Huang J, Liu T, Xu LG, et al. SIKE is an IKK epsilon/TBK1-associated suppressor of TLR3- and virus-triggered IRF-3 activation pathways. EMBO J 2005; 24:4018-4028.

24 Lin R, Yang L, Nakhaei P, et al. Negative regulation of the retinoic acid-inducible gene I-induced antiviral state by the ubiquitinediting protein A20. J Biol Chem 2006; 281:2095-2103.

25 Kayagaki N, Phung Q, Chan S, et al. DUBA: a deubiquitinase that regulates type I interferon production. Science 2007; 318:16281632.

26 Arimoto K, Takahashi H, Hishiki T, et al. Negative regulation of the RIG-I signaling by the ubiquitin ligase RNF125. Proc Natl Acad Sci USA 2007; 104:7500-7505.

27 Gack MU, Shin YC, Joo CH, et al. TRIM25 RING-finger E3 ubiquitin ligase is essential for RIG-I-mediated antiviral activity. Nature 2007; 446:916-920.

28 Yamanaka K, Ishikawa H, Megumi Y, et al. Identification of the ubiquitin-protein ligase that recognizes oxidized IRP2. Nat Cell Biol 2003; 5:336-340.

29 Ishikawa H, Kato M, Hori H, et al. Involvement of heme regulatory motif in heme-mediated ubiquitination and degradation of IRP2. Mol Cell 2005; 19:171-181.

30 Iwai K, Ishikawa H, Kirisako T. Identification, expression, and assay of an oxidation-specific ubiquitin ligase, HOIL-1. Methods Enzymol 2005; 398:256-271.

31 Cong YS, Yao YL, Yang WM, Kuzhandaivelu N, Seto E. The hepatitis B virus X-associated protein, XAP3, is a protein kinase C-binding protein. J Biol Chem 1997; 272:16482-16489.

32 Marin I, Ferrus A. Comparative genomics of the RBR family, including the Parkinson's disease-related gene parkin and the genes of the ariadne subfamily. Mol Biol Evol 2002; 19:20392050.

33 Bayle J, Lopez S, Iwai K, Dubreuil P, De Sepulveda P. The E3 ubiquitin ligase HOIL-1 induces the polyubiquitination and degradation of SOCS6 associated proteins. FEBS Lett 2006; 580:2609-2614.

34 Tian Y, Zhang Y, Zhong B, et al. RBCK1 negatively regulates tumor necrosis factor- and interleukin-1-triggered NF-kappaB activation by targeting TAB $2 / 3$ for degradation. J Biol Chem 2007; 282:16776-16782.

35 Kothapalli R, Nyland SB, Kusmartseva I, et al. Constitutive production of proinflammatory cytokines RANTES, MIP-1beta and IL-18 characterizes LGL leukemia. Int J Oncol 2005; 26:529535.

36 Bach JF. Infections and autoimmune diseases. J Autoimmun 2005; 25 Suppl:74-80.

37 Rotondi M, Chiovato L, Romagnani S, Serio M, Romagnani P. Role of chemokines in endocrine autoimmune diseases. Endocr Rev 2007; 28:492-520.

38 Mondini M, Vidali M, Airo P, et al. Role of the interferon-inducible gene IFI16 in the etiopathogenesis of systemic autoimmune disorders. Ann N Y Acad Sci 2007; 1110:47-56.

39 Lin R, Noyce RS, Collins SE, Everett RD, Mossman KL. The herpes simplex virus ICP0 RING finger domain inhibits IRF3and IRF7-mediated activation of interferon-stimulated genes. $J$ Virol 2004; 78:1675-1684.

40 Henderson G, Zhang Y, Jones C. The Bovine herpesvirus 1 gene encoding infected cell protein 0 (bICP0) can inhibit interferondependent transcription in the absence of other viral genes. $J$ Gen Virol 2005; 86:2697-2702.

41 Bibeau-Poirier A, Gravel SP, Clement JF, et al. Involvement of the IkappaB kinase (IKK)-related kinases tank-binding kinase $1 / \mathrm{KKKi}$ and cullin-based ubiquitin ligases in IFN regulatory factor-3 degradation. J Immunol 2006; 177:5059-5067.

42 Saitoh T, Tun-Kyi A, Ryo A, et al. Negative regulation of interferon-regulatory factor 3-dependent innate antiviral response by the prolyl isomerase Pin1. Nat Immunol 2006; 7:598-605.

43 Saira K, Zhou Y, Jones C. The infected cell protein 0 encoded by bovine herpesvirus 1 (bICP0) induces degradation of interferon response factor 3 and, consequently, inhibits beta interferon promoter activity. $J$ Virol 2007; 81:3077-3086.

44 Graff JW, Ewen J, Ettayebi K, Hardy ME. Zinc-binding domain of rotavirus NSP1 is required for proteasome-dependent degradation of IRF3 and autoregulatory NSP1 stability. J Gen Virol 2007; 88:613-620. 